\title{
Si devono innaffiare o annaffiare le piante?
}

\author{
Luisa di Valvasone
}

PUBBLICATO: 16 FEBBRAIO 2021

\section{Quesito:}

Sono giunti alla redazione alcuni quesiti in merito a quale sia la forma (più) corretta tra annaffiare e innaffiare e, di conseguenza, tra i derivati annaffiatoio e innaffiatoio.

\section{Si devono innaffiare o annaffiare le piante?}

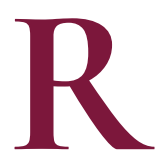

iguardo a quale sia la forma da preferire tra annaffiare e innaffiare, la risposta è semplice: si tratta di due varianti ed entrambe sono corrette. Lo stesso si può dire per i sostantivi derivati annaffiatoio e innaffiatoio, che si riferiscono entrambi al "recipiente con lungo beccuccio espanso e traforato all'estremità in modo che l'acqua ne spilli e cada come pioggia" (DevotoOli 2019). I dizionari moderni mettono a lemma entrambe le forme del verbo ma rimandano perlopiù (cosi come per i derivati) da innaffiare ad annaffiare, nel significato di "bagnare d'acqua sostituendo gli effetti della pioggia: annaffiare $i$ fiori, il giardino; annaffiare le strade, bagnarle a scopo di pulizia con l'autoinnaffiatrice", per estensione "bagnare completamente, infradiciare: il bambino mi ha annaffiato con la pistola ad acqua" e in senso figurato e scherzoso "annacquare, diluire: annaffiare il vino" o "accompagnare i cibi con una bevanda" (Devoto-Oli 20I9); il GDLI alla voce innaffiare registra inoltre la variante antica scempia inaffiare e alcune locuzioni e usi figurati come innaffiare di lacrime/di pianto, innaffiare col proprio sangue/col proprio sudore, innaffiare il giardino di una donna (possederla carnalmente, oggi in disuso) e innaffiarsi la gola.

Dal punto di vista etimologico i dizionari sono concordi nel presumere una comune derivazione delle due forme del verbo dal latino volgare *inafflāre, derivato di afflāre 'soffiare' con il prefisso locativo in-. Secondo l'Etimologico la forma annaffiare presenta la variante toscana an-del prefisso in-, oltre alla geminazione della nasale $n$ davanti alla vocale iniziale della base (a). Il DELI rimanda al LEI, secondo cui "la variazione inn-/ann-corrisponde a quella dell'it[aliano] a[ntico] innascondere/annascondere, che, nel caso di abscondere, risale a differenziazioni dialettali".

L'alternanza tra le due varianti è documentata già a partire dalle origini della nostra lingua. Innaffiare è la variante più vicina all'etimo latino e fa la sua prima comparsa in italiano nel volgarizzamento Della miseria dell'uomo di Bono Giamboni del I292 ("e feci orti e giardini [...] e feci vivai, acciò ch'io innaffiassi l'erbe e le piante fruttuose"). Il TLIO riporta per innaffiare anche diverse varianti come inafiato, innafiata, "naffiare e la scempia inaffiare, segnalata anche nel GDLI, e presente sia in esempi di autori toscani sia nell'unico esempio non toscano, bensi siciliano, riportato nella voce del TLIO. Annaffiare è invece datato nel DELI al I350 circa nel volgarizzamento di un'opera di Crescenzi, ma possiamo rintracciare nel TLIO un'altra attestazione nel Dittamondo di Fazio degli Uberti risalente agli anni I345-67 ("Vedi 'l paese che la Fame graffia / e donde l'Oreade già la tolse. / E come leggi in molte pataffia, / quest'e si fuor d'ogni dolce pastura, / che poco giova se pioggia l'annaffia"). Il sostantivo, in entrambe le forme, compare invece nell'italiano scritto a partire dal XVII secolo (cfr. l'Etimologico).

Le voci innaffiare e annaffiare fanno dunque il loro ingresso nella lingua scritta a distanza di poco più di cinquant'anni l'una dall'altra e continuano ad alternarsi nei secoli successivi, come dimostrano anche le ricche testimonianze riportate dal GDLI per entrambe le varianti: hanno scelto di impiegare 
la forma con an- autori come Bernardo Davanzati, Manzoni, Montale; mentre altri - Boccaccio, Ariosto, Pavese - hanno preferito innaffiare e i suoi derivati. Tuttavia sembrerebbe che a partire dal XVI secolo la variante con an- risulti la forma popolare più comune. Nel dizionario di Francesco Sansovino, Ortografia delle voci della lingua nostra, pubblicato a Venezia nel I568, si legge: "annaffiare è in uso del volgo, molto più chinnaffiare". Anche la prima edizione del Vocabolario degli Accademici della Crusca del I6r2 riporta alla voce innaffiare "oggi più comunemente annaffiare". Tale dicitura rimane e si amplia nella seconda (I623) e nella terza edizione (I69I) del Vocabolario con il riferimento ad annaffiatoio ("E 'l Vasello, con che s'annaffia, Annaffiatoio"), e a partire dalla seconda edizione anche annaffiare viene messo a lemma col significato di 'innaffiare'. La voce innaffiatoio compare invece solo a partire dalla quarta edizione (I729-38), ma nella quinta (I863-I923) si legge "più comunemente annaffiatoio".

La preferenza per la variante annaffiare è confermata anche dai più recenti strumenti lessicografici. Nel DOP la forma innaffiare è considerata variante meno comune di annaffiare, e lo stesso è indicato per la coppia di sostantivi annaffiatoio/innaffiatoio; la stessa indicazione si ritrova nei principali dizionari moderni (Zingarelli 2020, Devoto-Oli 2019, GRADIT, Vocabolario Treccani online) che, come già detto, alla voce innaffiare inseriscono un rinvio al lemma annaffiare.

Le occorrenze presenti nel corpus di italiano scritto DiaCORIS, che raccoglie scritti di diversi generi pubblicati a partire dal I86I, evidenziano una prevalenza minima delle varianti con an-: emergono infatti I7 occorrenze per annaffiare e I4 per innaffiare (a cui però possiamo aggiungerne 4 per la variante antica inaffiare, in testi risalenti ai primi anni del Novecento), 9 occorrenze per il sostantivo annaffiatoio e 4 per innaffiatoio. In alcuni casi l'alternanza tra le varianti si presenta anche all'interno di opere diverse di uno stesso autore, come ad esempio Pirandello:

Vedevo qualche sera nel terrazzino lì accanto la mammina di casa in veste da camera, intenta a innaffiare i vasi di fiori. "Ecco la vita!" pensavo. (Luigi Pirandello, Il fu Mattia Pascal, 1904)

Corrado Selmi, gravato dal peso di Roberto, stette un po' a guardare i cenni del Passalacqua, che seguitava a implorar carità per il cuore malato della sua povera moglie, carità per Roberto così perduto, carità per la casa che sarebbe andata a soqquadro; e scattò alla fine, scrollandosi, in una risata pazzesca:

- Ma da' qui! - disse, ghermendo l'annaffiatojo e avviandosi di furia al terrazzo.

- Ma che facciamo sul serio? Annaffiavi? E seguitiamo ad annaffiare! Qua... qua... così! così! Pioggia, Olindo! pioggia! pioggia! (Luigi Pirandello, I vecchi e i giovani, 1909)

Per quanto riguarda l'ambito letterario, le ricerche, relative alle forme allinfinito dei verbi, nel PTLLIN - il cui corpus è composto dai romanzi vincitori del Premio Strega a partire dal 1947 fino al 2007 e da una quarantina di altri romanzi italiani - evidenziano una preferenza per la variante innaffiare (Io occorrenze in otto opere della forma all'infinito, nessuna per la variante antica inaffiare) rispetto ad annaffiare (4 occorrenze).

Le ricerche in rete restituiscono un quadro leggermente diverso, confermando però l'attuale vitalità di tutte le varianti: il 30/9/2020 nelle pagine in italiano di Google emergono 380.0oo risultati per la forma annaffiare (282.00o per annaffiatoio) e 406.00o risultati perinnaffiare (ma 95.60o per innaffiatoio), su Google libri risultano invece 24.Ioo risultati per annaffiare (Io.20o per annaffiatoio) e 33.0oo risultati per innaffiare (8.430 per innaffiatoio).

I contesti in rete e nei corpora non evidenziano una differenziazione nell'uso odierno delle varianti né di tipo semantico né di tipo geografico; i dizionari non accennano a differenze regionali e neanche 
l'AIS Atlante linguistico ed etnografico dell'Italia e della Svizzera meridionale, ci viene in aiuto: all'interno della carta I425, dedicata al verbo irrigare, troviamo due sole attestazioni di annaffiare e innaffiare in due località, entrambe in Toscana. I quesiti giunti alla nostra redazione provengono da diverse località d'Italia - Trieste, Milano, Roma, Orta di Atella (in provincia di Caserta), Serrenti (in Sardegna meridionale) - e di questi solo nel quesito del lettore di Serrenti è espressa la preferenza d'uso per una delle due varianti (in questo caso innaffiare: "Da almeno quarant'anni e, se tutto procederà bene, per altrettanti anni, l'atto del mettere acqua a piante e fiori, a casa mia è quello dell'innaffiare").

Sembrerebbe dunque che non vi siano restrizioni o differenze d'uso né per l'una né per l'altra variante (e lo stesso per i derivati), anche se ricordiamo che gli strumenti lessicografici segnalano la forma annaffiare come più comune rispetto a innaffiare. Scegliete quindi liberamente se annaffiare o innaffiare le vostre piante.

\section{Cita come:}

Luisa di Valvasone, Si devono innaffiare o annaffiare le piante?, "Italiano digitale", 2021, XVI, 2021/1 (gennaio-marzo)

DOI: $10.35948 / 2532-9006 / 2021.5476$

Copyright 2021 Accademia della Crusca

Pubblicato con licenza creative commons CC BY-NC-ND 